\title{
EPIDEMIA Y SOCIEDAD \\ en el Valle de La Convención, 1932
}

Marcos Cueto*

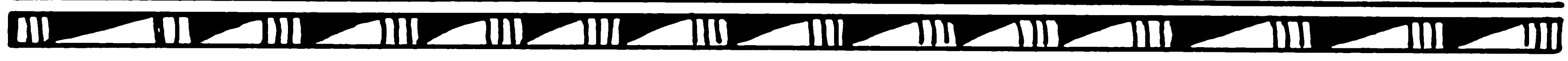

Cuando la carretera llegó a Quillabamba, a fines de 1932, sus habitantes no pudieron dejar de celebrarlo con un baile en los salones de la casa-hacienda Macamango. Seguramente algunos bailaron pensando que era la última vez que lo hacían. No porque no hubiese nada que festejar en el futuro de una localidad en progreso, sino porque también había llegado a la ciudad una terrible epidemia de malaria. Ese día, como una metáfora de modemidad y muerte, los que festejaban la llegada de la carretera, bailaban en el mismo edificio en cuyo sótano se habían refugiado ya las primeras víctimas de la epidemia ${ }^{1}$.

Así empezó una de las epidemias más feroces que azotaron al sur andino durante la época republicana. Sus

- Este artículo forma parte de una investigación más amplia titulada "Epidemias, Políticas Sanitarias y Reacciones Sociales en el Perú: 1900-1980", que se llevó a cabo con el auspicio de Fomciencias, Lima, y Social Science Research Council, Nueva York.

1 Córtez, (1938: 5). 
terribles consecuencias, frecuentemente mencionadas pero aún poco estudiadas, nos recuerdan que la enfermedad generalizada ha sido una frecuente ocurrencia en la historia del Perú. Después de la conquista, los Andes fueron el escenario de violentas epidemias de tifus, viruela y otros males que diezmaron significativamente la población ${ }^{2}$. Aunque de dimensiones más circunscritas que las epidemias del período colonial, la que empezó en 1932 fue suficiente para revelar, la absoluta insuficiencia de los servicios sanitarios oficiales, la contradicción inhumana entre los sistemas de colonización de la selva alta y la salud pública, y la terca persistencia de creencias, valores y hábitos indígenas con respecto a la salud, la enfermedad y la muerte.

\section{UNA GRAN MORTANDAD}

Quillabamba era la capital de la provincia de La Convención, un territorio formado por la hoya del rio Urubamba, que con una extensión de 105,122 kilómetros cuadrados, constituía uno de los valles más extensos y menos poblados no sólo del departamento del Cusco sino de todo el Perú. Desde comienzos del siglo XX la región había experimentado una repentina prosperidad gracias a su inserción al mercado cusqueño como abastecedora de coca, azúcar, viveres y otros productos agrícolas. La construcción en 1927 del ferrocarril del Cusco a Machupichu, incrementó el flujo de migrantes que se movilizaban a estas tierras para trabajar en alguna de las recientemente creadas haciendas. Sin embargo, pocos prestaron atención a los peligros que ofrecía el rio Urubamba, una voz quechua que quería decir "tierras de insectos"3.

2 Véase Dobyns (1963:) y Cook (1981).

3 Paz Soldán, (1877: 966). 
La epidemia comenzó en uno de los extremos de la explotación de la selva. En agosto de 1932 aparecieron los primeros casos en la localidad de Siriato, dentro del territorio de las tribus Machiguengas y no lejos de las misiones dominicas del Koribeni. Aunque no se conoce con certeza su origen, probablemente la enfermedad llegó de las selvas del Brasil. La epidemia fue avanzando rio arriba hasta llegar a Quellounu, y luego se fue bifurcando en dos direcciones; un brazo de la epidemia remontó el río Urubamba, afectando el valle de La Convención, mientras que otro siguio, en sentido contrario, el curso del rio Yanatile, infectando el valle de Lares, en la provincia de Calca.

Alarmados por la rápida expansión de la enfermedad, el 7 de noviembre de 1932, el obispo, el alcalde y otras autoridades de Quillabamba se reunieron en el local del Concejo Provincial para pedir ayuda a la ciudad del Cusco. En su mensaje notificaron que el vecindario estaba dejando la ciudad por una "epidemia desconocida que causaba una gran mortandad"4. Pocos días después, en un recorrido por la provincia, el doctor Samuel Geller, médico titular de La Convención, determinó que el distrito más afectado era el de Echerate, ubicado a sólo 35 kilómetros de Quillabamba5. Las primeras noticias que empezaron a difundir los diarios de la ciudad del Cusco, fueron aterradoras:

Vecinos llegados hace poco de los centros poblados de Sahuayaco, Chacanares, Chaco, Roroncoe y Sajiruyo informan [...] que los enfermos se cuentan en cada caserio por decenas; que los

4 "Quillabamba es azotada por una epidemia" El Sol (Cusco) (de ahora en adelante ES (C) Noviembre 8, 1932, p. 2.

5 Samuel Geller a Prefecto del Cuzco, Noviembre 14, 1932. Prefectura, Comunicaciones Recibidas, Legajo 7, Año 1932. Archivo Departamental del Cuzco (de ahora en adelante $A D C$ ). 


\section{Marcos Cueto}

parientes de los atacados temiendo el contagio emigran en todas direcciones en éxodo interminable, abandonando a sus familiares a su propia suerte. Los ranchos de esta infeliz gente quedan abandonados dejando a los cadáveres insepultos que son comidos por los cuervos ${ }^{6}$.

Las noticias de cuerpos abandonados se extendieron como un reguero de pólvora y fueron confirmadas por testimonios posteriores ${ }^{7}$. Al parecer, la dispersión y el aislamiento de las viviendas, la escasez de las vías de comunicación y los precarios recursos médicos impidieron atender a muchas familias. En el caso que todos sus miembros enfermaban, no había nadie capaz de pedir ayuda o dar sepultura a los muertos ${ }^{8}$.

La malaria era conocida en La Convención. La última epidemia, inmediatamente anterior a la de 1932, había ocurrido entre 1898 y 1902. Sin embargo, según las autoridades de la región, ninguna había sido tan fuerte

\section{2 , p. 2.}

6 "Correspondencia de La Convención", ES (C) Noviembre 24,

7 Un médico que estuvo trabajando durante la epidemia corroboró la versión de víctimas dejadas a su propia suerte: "los gallinazos habian dado cuenta de los cadáveres de muchos individuos muertos en absoluto abandono. $Y$ el hecho es fácil de explicarse si se tiene en cuenta que un gran porcentaje de la peonada vive no en caserios más - menos compactos, si no en casuchas aisladas (chozas) distantes unas de otras varios kilómetros". Córtez (1938:10). Años después un recopilador del folklore recogió el testimonio de una maestra que recordaba la epidemia de 1932 donde: "los perros daban cuenta de los cadáveres", Valencia Vargas, (1961:33).

8 La desesperación permitió que se desarrollase un gran fervor religioso. Los Dominicos organizaron por las calles de Quillabamba triduos (procesiones de tres días) donde se hacian penitencias y elevaban plegarias. Los fieles iban escoltando las imágenes con: "cruces, de ellas chicas, de ellas grandes, cantando tristes endechas en quechua y castellano, que hieren los corazones con sus letras de arrepentimiento $y$ perdón". Menendez Rua (1948: 258). 
"ni tan rebelde y de difícil curación", como la epidemia que estaban experimentando al comenzar la década del treinta ${ }^{9}$. El cuadro 9 sugiere que los niños fueron los más afectados y nos da una idea del número de personas enfermas en Vilcabamba y Santa Ana, dos de los distritos más poblados de la provincia de La Convención.

\section{CUADRO № 1}

MoRbilidad de Malaria en Vilcabamba y Santa ANa

\begin{tabular}{lrrrc}
\hline Localidad & $\begin{array}{c}\text { Menores de } \\
\text { diez años }\end{array}$ & $\begin{array}{c}\text { Porcentaje } \\
\text { de enfermos }\end{array}$ & $\begin{array}{c}\text { Mayores de } \\
\text { diez años }\end{array}$ & $\begin{array}{c}\text { Porcentaje } \\
\text { de enfermos }\end{array}$ \\
\hline Santa Teresa & 39 & 82 & 82 & 59 \\
Hda. Huadquiña & 63 & 78 & 230 & 70 \\
Quellomayo & 0 & 0 & 59 & 81 \\
Hda. Santa Rosa & 47 & 60 & 153 & 54 \\
Santa María & 12 & 91 & 53 & 89 \\
Hda. Paltaibamba & & & & \\
y anexos & 134 & 81 & 302 & 83 \\
Hda. Santa Ana & 42 & 100 & 91 & 84 \\
Hda. Macamango & 29 & 63 & 95 & 59 \\
Hda. Potrero & 159 & 91 & 325 & 85 \\
Hda. Pintobamba & 205 & 94 & 366 & 86 \\
Hda. Uchumayo & 155 & 91 & 325 & 72 \\
Hda. Mazapata & 33 & 78 & 73 & 57 \\
Hda. Urpipata & & & & \\
alta y baja & 10 & 88 & 24 & 79 \\
Quillabamba & 342 & 81 & 754 & 66 \\
\hline Total & 1,270 & 82 & 2,932 & 74 \\
\hline Fut & & & &
\end{tabular}

Fuente: Ugarte y Geller (1933: 530).

Los porcentajes han sido redondeados.

Las seis primeras localidades pertenecen al distrito de Vilcabamba, las ocho siguientes al de Santa Ana.

9 "Manifiesto de las fuerzas vivas de La Convención y Lares sobre la epidemia y medidas que urge adoptar", El Comercio (Lima) Setiembre 11, 1933, p. 4. 


\section{Marcos Cueto}

La despoblación que empezó a producir la epidemia en el valle resultó no tan sólo de la enfermedad inisma sino de sus secuelas: la falta de trabajo y el hambre generalizado. Según un testimonio el panorama era desolador: "terrenos de cultivo se vuelven bosques donde se pasean animales salvajes y los domésticos salvajizados"10. Los precios del ganado y de otros productos de la región que se vendían en el Cusco bajaron notablemente por el temor equivocado de muchas personas de verse infectadas ${ }^{11}$. Otro efecto de la epidemia fue la venta de muchas haciendas a precios irrisorios, lo que posiblemente favoreció una mayor concentración de la propiedad rural en el valle $e^{12}$.

Como los enfermos morian en 24 horas, con vómitos, fiebre alta, fuertes dolores de cabeza y el semblante pálido, se pensó en un primer momento que la epidemia era de fiebre amarilla ${ }^{13}$. La forma más común de malaria conocida en el Perú se caracterizaba por fiebres intermitentes que debilitaban el organismo, pero no mataban de una manera fulminante. Aunque al poco tiempo de comenzada la enfermedad, el médico titular de la provincia se inclinó por un diagnóstico clínico en favor de la malaria, sólo muestras entomológicas y hematológicas podrían mostrar incontestablemente la identidad de la enfermedad. Es decir, había que detectar la presencia de mosquitos del género Anopheles, identificado por el inglés Ross en 1986, y encontrar en la sangre de los enfermos

10 "La verdad sobre el desarrollo del paludismo en el valle de Lares. Una carta interesante de los hacendados de esa región". El Comercio (Cuzco) Noviembre 21, 1933, p. 2.

$"$ Ardiles Villegas (1983: 90).

12 Florez Muñiz (1946).

13 Carlos Monge Medrano, "La epidemia de los valles de La Convención y Lares", ES (C) Octubre 17, 1933, p. 2. Aunque Monge no compartió este punto de vista menciona que otros doctores si creyeron que la epidemia fue fiebre amarilla. 
el parásito Plasmodium, un hematozoario aislado por el francés Laveran en $1880^{14}$.

Tanto el trabajo de Ross como el de Laveran habían establecido que era absolutamente necesaria la presencia del mosquito en la transmisión de la malaria. La descripción del proceso es la siguiente: el parásito de la malaria atraviesa en su vida por dos períodos; un ciclo asexual que se cumple en el hombre y uno sexual que se produce en el mosquito. En la etapa asexual, el parásito es introducido en la sangre de una persona en la secreción de saliva que deja la picadura de un mosquito hembra (la sangre es un elemento esencial para la maduración de sus huevos). Una vez en la sangre humana el parásito se cobija en algunos glóbulos rojos provocando su ruptura lo que es la causa de los escalofrios típicos de la malaria. Algunos parásitos evolucionan hacia formas sexuales llamadas gametos $y$, cuando un mosquito no infectado chupa la sangre de esta persona, vuelven al estómago del Anopheles donde algunos gametos machos fertilizaban a las hembras. Posteriormente, las células fertilizadas llegan a las glándulas salivales del mosquito. $\mathrm{Si}$ este mosquito pica a una persona sana le transmite la malaria ${ }^{15}$.

Luis Angel Ugarte, el médico sanitario departamenal del Cusco, efectuó estudios tratando de identificar a los mosquitos de la región sin lograr encontrar el Aedes aegypti, el vector de la fiebre amarilla. En cambio, cuando Ugarte envió muestras al Instituto Oswaldo Cruz de Rio de Janeiro, éstas fueron clasificadas como Anopheles pseudopunctipennis y Anopheles argyrotarsis ${ }^{16}$. Por otro

14 Para una visión histórica de los conocimientos sobre la malaria, véase Harrison, (1978).

15 Una descripción más detallada del proceso aparece en: Martin

D. Young, (1976: 353-396).

16 Willie, (1934: 645). 


\section{Marcos Cueto}

lado, un científico extranjero recibió el encargo de hacer un estudio entomológico y encontró el Anopheles pseudopunctipennis, considerándolo el principal, si no el único, agente transmisor del hematozoario de Laveran en la región ${ }^{17}$.

Aunque nunca llegó a ser muy explícito en los estudios que realizo, Ugarte afirmó que los exámenes de sangre revelaron la presencia del "germen del paludismo especialmente de la especie denominada Plasmodium falciparum, agente de la malaria maligna o tropical, y también algunos de paludismo benigno"18. (Paludismo era el otro nombre con el que popularmente se conocía a la enfermedad de la malaria). De las tres formas en las que se presenta el parásito Plasmodium en el Perú: malarie, vivax y falciparum, esta última es la más infrecuente y la única que causa una muerte fulminante.

En realidad nunca hubo un estudio detallado para precisar que se trata de una epidemia de malaria. La prueba definitiva la hubiese dado una prueba microbiológica que permitiera encontrar el parásito en la sangre de los pacientes atacados. Otro criterio importante para determinar la presencia de falciparum hubiese sido el examen clínico, que en estos casos presenta lesiones cerebrales y parálisis. Tanto las pruebas hematológicas como las descripciones clínicas fueron vagas $y$ no permite hacer un diagnóstico concluyente en favor de la malaria. Sin embargo, el criterio epidemiológico, es decir, la gran y fulminante mortandad, sumada a la presencia comprobada de Anopheles, hacen plausible creer que efectivamente se trata de una malaria maligna causada por el Plasmodium falciparum.

La falta de mayor determinación sobre la identidad de la enfermedad se debió también a que durante los

17 lbid.

18 Luis Angel Ugarte, "De Sanidad, sobre la epidemia de los valles de La Convención y Lares", ES (C) Noviembre 29, 1932, p. 2. 


\section{Epidemia y Sociedad en el Valle de La Convención}

primeros momentos de la epidemia hubo poco tiempo para este tipo de discusiones. La epidemia avanzaba rápidamente remontando el curso de los ríos, pasando de territorios de menor altitud hasta llegar a las estribaciones de los Andes. Más allá de una altura de 2,500 metros sobre el nivel del mar podian presentarse algunos casos (personas picadas por mosquitos en altitudes menores), pero estos no transmitian la enfermedad por las dificultades de sobrevivencia del Anopheles a esa altitud. Existió una relación inversamente proporcional entre la epidemia y la altura de una región. Conforme se iba descendiendo hacía la selva aumentaba el número de casos, siendo estos menores en las haciendas más altas del valle.

Este peculiar curso se explica en parte por las mejores condiciones climáticas que ofrecía la selva baja y húmeda para el crecimiento de los Anopheles. El caudoloso rio Urubamba, de caprichosas ondulaciones, piedras enormes y playas pequeñas, daba origen en tiempos de sequía a charcos, brazos estancados y pequeñas lagunas conocidas en la región como cochas, lugares donde proliferaban las larvas de los mosquitos ${ }^{19}$. Los criaderos de larvas se encontraban en cualquier agua empozada, ya sea clara o turbia, con o sin vegetación ${ }^{20}$. La cantidad de criaderos que existian y su proximidad a las viviendas humanas incrementaban el peligro.

La enfermedad se difundía también porque la gente enferma se volvía nómada y huía de sus lugares de residencia, tratando de llegar a lugares donde no existiese el mal, sin saber que lo estaba difundiendo. El factor de movilidad fue más decisivo, si se toma en cuenta que los primeros casos eran de personas que se internaban a lugares infectados de la selva y volvian a centros poblados. Ello hace suponer que en la difusión de la enferme- 


\section{Marcos Cueto}

dad jugaron un rol importante los arrieros que frecuentemente trajinaban el valle con sus bestias de carga siguiendo el curso de los rios ${ }^{21}$. En estos viajes no sólo llevaban productos sino a otros trabajadores de la sierra, generalmente de mayor suceptibilidad a la malaria.

Nadie en Quillabamba estaba preparado para combatir una epidemia de éstas dimensiones. Las madres misioneras dominicas improvisaron un hospital utilizando las instalaciones de una escuela ${ }^{22}$. En realidad la concentración de los enfermos en un sólo centro hospitalario en Quillabamba fue una medida contraproducente. Si se toma en cuenta que era imposible proteger adecuadamente a estos enfermos de la picadura de los Anopheles y estando estos en medio de un centro poblado donde transitaban muchas personas sanas, es plausible suponer que esta concentración fue uno de los factores que permitio que la epidemia se transmitiese rápidamente.

En general, todo el departamento del Cusco tenía serias deficiencias sanitarias. Por ejemplo, en este departamento sólo habian doce médicos titulares y un hospital con una capacidad máxima de 200 camas para una población que sólo en la capital se calculaba en 30,000 habitantes ${ }^{23}$. Un médico de Lima calculaba las necesidades de la población del departamento en, por lo menos, dos mil camas $^{24}$. Asimismo, aunque la Sociedad de Beneficencia Pública del Cusco existía desde el siglo diecinueve, en los años treinta se encontraba atravesando una profunda

21 Según un autor el arrieraje con su movilidad intensa estaba intimamente ligado a la epidemia, Paz Soldán, (1933).

22 Desde mediados de noviembre de 1932 hasta diciembre de 1934 se atendieron en este hospital 2,770 enfermos de malaria. Menendez Rua (1948: 258).

23 La estimación de la población de la ciudad fue realizada en 1930 y corresponde al viajero K. Grub, véase Tamayo Herrera (1980: 129).

24 Paz Soldán, (1933: 12). 


\section{Epidemia y SOCIedad en el Valle de La Convención}

crisis. Según el médico titular de La Convención, la mayor parte de las defunciones durante la epidemia se debieron a la falta absoluta de atención a los enfer$\operatorname{mos}^{25}$.

La malaria actuó como un factor desencadenante de otras enfermedades. Aquellos que sobrevivían a la malaria quedaban tan débiles que eran generalmente víctimas indefensas de la tuberculosis, la viruela y de otros males no menos mortales. El abandono de cadáveres, sumado a las precarias condiciones de atención, llevó a un médico de Lima a decir de los pobladores de la región que "no están preparados ni siquiera para morir"26.

Un comentario fechado en 1933 nos da una clave de quienes eran los menos preparados para enfrentarse a los rigores de la enfermedad: "el mal hace más víctimas en los indios del valle que en los salvajes machiguengas, y más entre los procedentes de las punas, que en los de los valles"27. Esto se explica porque los hombres y mujeres de las tribus nativas habian adquirido cierta inmunidad a la enfermedad al vivir en un ambiente cargado de Anopheles. La mayoría había sufrido, durante la niñez, ataques leves de malaria y los que sobrevivían quedaban inmunes al mal. Asimismo ellos no representaban mayor peligro de extensión de la enfermedad, puesto que tenían poco contacto con poblaciones exteriores a si mismas. Los migrantes, en cambio, provenían de las zonas medias $y$ altas de los Andes, lugares donde no existía la malaria, y por ello eran más suceptibles. Sin embargo, para acabar de entender las diferencias sociales que reveló la epidemia, es necesario reseñar el contexto económico de la región atacada.

\footnotetext{
25 Ugarte, "De Sanidad", p. 2.

26 Paz Soldán, (1933: 7).

27 "Manifiesto de las fuerzas vivas", p. 4.
} 


\section{Marcos Cueto}

\section{UN SISTEMA DE COLONIZACIÓN SIN SALUD}

A partir de la consolidación de un circuito lanero en la región sur del país a fines del siglo diecinueve, ciudades como Arequipa, Puno y Cusco cobraron nueva vida, jugando cada una de ellas un rol diferente y complementario ${ }^{28}$. Mientras que en la primera florecían las casas comerciales, en la segunda crecía el número de hacendados ganaderos. Por su lado, la agricultura del departamento del Cusco empezó a convertirse en la zona de abastecimiento de frutas y viveres para buena parte del sur del Perú. La mayoría de ellos eran producidos en la zona del alto Urubamba, una región hasta entonces casi abandonada por la civilización pero que, a partir de 1909, con la Ley de Tierras de Montaña que propició la concentración de la propiedad rural, experimentó un notable crecimiento del número de haciendas que enviaban sus productos a la ciudad del $\mathrm{Cusco}^{29}$.

En términos de su valor, los productos que rendian mayores ingresos a la región eran la coca, el azúcar y el aguardiente de caña ${ }^{30}$. Conforme las casas comerciales arequipeñas establecían vínculos más directos con las regiones laneras de Puno, las casas comerciales del Cusco se dedicaron a la redistribución de los productos de la ceja de selva y de la selva del departamento ${ }^{31}$. Según un estimado, los cultivos del valle de La Convención en el momento de la epidemia estaban repartidos de la siguiente forma:

28 Con respecto a las caracteristicas económicas de esta región, véase Jacobsen (1983: 89-148).

29 Fioravanti, (1976: 17).

30 Hobsbawm (1969: 35). El autor se refiere a la situación en 1915.

31 Brisseau, (1983: 23). 
Epidemia y Sociedad en el Valle de La Convencion

CUADRO № 2

Cultivos en el valle de La Convención

\begin{tabular}{lrr}
\hline Producto & Hectáreas & $\%$ \\
\hline Caña de azúcar & 800 & 11.9 \\
Coca en las haciendas & 800 & 11.9 \\
Coca en arriendos & 2,000 & 29.8 \\
Café & 400 & 5.9 \\
Cacao & 700 & 10.4 \\
Viveres (yucas, plátanos, & 2,000 & 29.8 \\
maíz, etc.) & 6,700 & 100.0 \\
\hline Total & &
\end{tabular}

Fuente: Ugarte y Geller, (1933: 511-512).

La creciente demanda por estos productos provocó un proceso de expansión de la superficie de las haciendas $y$ de concentración de la propiedad rural en pocas manos. No hubo ninguna diversificación o intensificación de los cultivos de las unidades productivas que generalmente se especializaban en uno o dos productos. La mayoría eran haciendas de reciente formación con propietarios ausentes que vivian en la ciudad del Cusco y que, con poco capital y menos posibilidades de conseguir mano de obra, habían iniciado la colonización de la selva con métodos primitivos.

Las difíciles condiciones del trabajo agrícola de tierras, no sólo incultas sino ubicadas entre quebradas estrechas, la resistencia de las tribus Machiguengas para convertirse en peones agrícolas y la distancia de los centros poblados hicieron difícil la creación de un mercado libre de trabajo o la utilización del sistema de enganche, utilizado en las haciendas de la costa peruana. Los hacendados de La Convención resolvieron el problema de 
la escasez de mano de obra promoviendo el establecimiento en la región de campesinos-colonos, un sistema menos costoso pero que rápidamente degeneró en una forma de dependencia personal.

E] hacendado era propietario de una gran extensión de tierras que no trabajaba directamente, sino que alquilaba a los así llamados arrendieres a cambio de una serie de obligaciones que incluían el servicio personal en las tierras y en la casa del propietario. La forma inicial de retener a los allegados en el valle era haciéndoles firmar un contrato con el hacendado, casi siempre por nueve años. Según el acuerdo, el alquiler del primer año no era pagado, pues se invertía en preparar el terreno para el trabajo agricola. Conforme aumentaba la calidad del terreno, se incrementaba la cantidad que recibía el hacendado por concepto de alquiler ${ }^{32}$.

Los arrendires, a su vez, subarrendaban pequeñas parcelas de tierra a los allegados a cambio de servicios $y$ productos $^{33}$. Generalmente los arrendires cumplian las obligaciones personales con el hacendado a través de los allegados. Estas obligaciones incluian la de trabajar un número determinado de días del mes en las labores agrícolas del propietario, enviar un sirviente a la casa del patrón y proveer jomaleros que trabajen 15 dias al año en las tierras del hacendado, recibiendo a cambio salarios infimos o sólo agua y comida.

En la última escala social de este complicado sistema de colonización estaban los habilitados o peones agricolas, quienes no poseían tierra y trabajaban por breves temporadas en la hacienda, en las tierras del

32 Cuadros (1949).

33 Este complejo sistema perduró hasta comienzos de los años sesenta y ha sido analizado extensamente en Fioravanti (1976). Asimismo es de interés consultar las relaciones que se establecen entre este sistema de explotación agrícola y la sanidad en: Kuczynski Godard (1945: 79). 


\section{Epidemia y SOCIedad en el Valle de La Convencion}

arrendire o en las del allegado. Asimismo, había una población flotante de comerciantes, arrieros y mercachifles que iban y venían de la región, llevando productos al Cusco.

Tanto los allegados como los arrendieres y los habilitados eran generalmente indios de las sierras de Cusco, Apurimac, Puno, Arequipa y Ayacucho, que llegaban al valle por la falta de tierras en sus regiones de origen. La existencia de una nueva población no inmune a la malaria fue uno de los factores fundamentales para explicar la alta mortalidad. Estos migrantes serranos, desnutridos por la miseria que creaba el sistema de trabajo rural $e$ indefensos ante los nuevos peligros ambientales, fueron las principales víctimas de la epidemia. Ello se explica porque, en términos generales, la morbilidad y la mortalidad era mucho más elevada entre los indígenas, y "especialmente en lo arriendos" 34 .

En 1944 un estudiante de la Universidad del Cusco visitó Quellomayo, un poblado cercano a la hacienda Huadquiña, y describió una escena dentro de una vivienda de arrendires que no debe haber sido muy diferente de lo que ocurrió en muchas chozas durante 1932:

Entrando en esta vivienda, nos sobrecojemos de espanto y miedo ante la muerte, ya que a la vista se nos presentan dos o tres hombres estirados en el lecho de dolor teniendo por cama un sólo poncho, hombres que se quejan y están tiritando, hasta el extremo de oirse el entrechocar de los dientes [...] Se hallan tan debilitados y sin aliento alguno estos enfermos, que no tienen fuerzas para mirar ni para ver quién es la persona que acaba de entrar [...] rostros pálidos, amarillentos, 
exángues [...] en una palabra parecen expectros humanos. Si le preguntamos que tiene, a entrecortadas y débiles voces nos responde: "me esta sacudiendo el paludismo"35.

Según un autor, el sistema de poblamiento que promovieron las haciendas en la zona fue una respuesta económica ineficiente al problema de la expansión comercial en la selva, sobre todo por la carencia total de una infraestructura social, sanitaria y educativa, que respaldara y consolidara el esfuerzo de colonización ${ }^{36}$. El abandono de los servicios educativos en la provincia se refleja, por ejemplo, en el hecho que recién en 1925 se dictaban sólo en Quillabamba los cinco años de primaria37. Otro indicador sugerente es que en 1936 en el distrito de Echerate sólo el 17 por ciento de los niños en edad escolar asistian a la escuela y el 80 por ciento de la población del distrito era analfabeta ${ }^{38}$.

La misma colonización de la selva había jugado un rol en el origen de la epidemia. Era habitual entre los campesinos y los hacendados quemar el bosque para hacer cultivable la selva, un procedimiento conocido con el nombre de "rose". La desforestación es conocida en el mundo como una de las causas más frecuentes del incremento de la malaria ${ }^{39}$. La destrucción de los bosques para permitir la explotación agrícola, alteró el habitat natural de los mosquitos incrementando su virulencia ${ }^{40}$. Asimismo la construcción de canales y carreteras y el establecimiento de nuevos cultivos que demandaban una

Vergara (1947).

5 Moscoso, (1944).

36 Hobsbawm (1969: 48).

37 Para una discusión del atraso educativo de la región, véase

38 Aragón Saravia (1941: 26-27).

39 Ackerknecht (1965: 94).

40 Paz Soldán (1933: 5). 
gran cantidad de riego, incrementaron las posibilidades de una epidemia de malaria. En resumen, la alteración del equilibrio ecológico y la presencia de una población no indemne desataron la epidemia, mientras que la gravedad que la situación revistió fue determinada por la miseria física a que se veían sometidos la mayoría de los habitantes del valle.

El panorama de abandono y pauperismo que imperaba en la región no dejó de ser censurado por varios médicos que visitaron el valle antes y después de la epidemia ${ }^{41}$. Un editorial de una revista médica de Lima, escrito apenas iniciada la epidemia, no se resistió de condenar en un tono religioso las acciones humanas que habian permitido que la epidemia se desarrolle. Al hacerlo reflejó el tremendo impacto que habian tenido los acontecimientos en ciertos sectores de la opinión pública nacional:

aquí se trata de uno de esos fenómenos epidemiológicos, $\tan$ fatales como las leyes cósmicas, que acontecen cuando se desdeñan las enseñanzas de la higiene. Las selvas tórridas del Cusco, colonizadas y explotadas en forma empírica sin la menor preocupación médico-social y sin rodear a los colonos de las seguridades mínimas necesarias a su existencia, se vengan cruelmente de tales errores arrojando de su seno a los que alli habían osado penetrar sin las debidas preocupaciones. La malaria es entonces, el angel bíblico que arroja del paraíso al hombre que delinque. $Y$ se ha delinquido porque cuando se explota la tierra y el trabajo humano, egoista y torpemente, no hay

1 Según un investigador médico, el sistema de colonización en La Convención "por esencia resistía un saneamiento duradero". Kuzcynski Godard (1945: 79). 
cultivo, si no profanación, que la fiebre castiga en forma aterradora y cuya aparición nadie, ni nada detiene ${ }^{42}$.

Pareceria una tarea imposible el tratar de combatir una epidemia en condiciones tan adversas. Sin embargo, desde el primer momento la población de la región hizo esfuerzos denodados por comprender y controlar la enfermedad. Algunas de las primeras medidas que intentaron las autoridades políticas y médicas de Quillabamba fueron: el aislamiento de los enfermos para tratar de evitar que fueran picados por los mosquitos, la administración diaria de quinina a los atacados, la protección de las viviendas con telas metálicas y mosquiteros, y la destrucción de las larvas de los Anopheles drenando o secando los pantanos y cualquier depósito de agua estancada ${ }^{43}$.

Sin embargo, poco es lo que pudo hacerse con los exiguos recursos locales asignados que apenas ascendían a 750 soles. Un médico estimaba el costo de la campaña, solamente en relación a las telas metálicas $y$ los mosquiteros, en 33,600 soles ${ }^{44}$. Para agravar aún más la situación, era muy difícil entonces encontrar en el Cusco el remedio por excelencia contra la malaria: la quinina $^{45}$. Estas dificultades llevaron a algunas autoridades políticas cusqueñas a exigir una pronta intervención del gobiemo central.

12 Anónimo (1933: 172).

43 "¿Por qué no desaparece el paludismo en los valles de La Convención y Lares", ES (C) Enero 5, 1933, p. 2.

44 El costo total de la campaña fue calculado en 56,300 soles, es decir que la mayor parte del gasto quiso ser destinado para una medida que posteriormente se reveló ineficaz. Ibid.

45 En general había disminuido la cantidad de quinina que ingresaba al país. Entre 1923 y 1927 se importó 28,199 kilos de quinina, mientras que durante el periodo 1928 a 1932 sólo 12,758. Paz Soldán (1933: 6). 


\section{LA POLITICA SANITARIA Y LAS REACCIONES SOCIALES}

A pesar que desde el mes de noviembre de 1932 se recibió en Lima la noticia de la epidemia de malaria en el valle de La Convención, la respuesta del gobierno se demoró varios meses. Esta tardanza puede explicarse por el estado incipiente de una organización sanitaria nacional que tenía un claro sesgo urbano y asistencialista. Hasta comienzos del siglo XX las municipalidades estuvieron a cargo de la sanidad en sus localidades. En 1903 se experimentó un cambio al establecerse una Dirección de Salubridad Pública que dependía del Ministerio de Fomento, una entidad que en 1935 fue reemplazada por el Ministerio de Salud. Esta asociación entre el Estado y la medicina oficial nació por el interés de los gobernantes de comienzos de siglo que vieron en la sanidad una manera de evitar las cuarentenas de los puertos, atraer al capital y a los inmigrantes europeos y ampliar el radio de acción del Estado en la sociedad ${ }^{46}$.

La Dirección de Salubridad era dirigida por clíni$\cos$ y cirujanos que manejaban la organización vertical y centralizadamente con poco conocimiento de la epidemiología social. Entre los médicos y las autoridades políticas en general, había poco interés por la salud pública, la medicina preventiva $y$ menos aún si se trataba de las provincias alejadas de los Andes. A las provincias del país eran enviados los asi llamados médicos titulares que atendían como podían, con precarios recursos, sueldos bajos y la inseguridad que un cambio de directores en Lima los relevase de su puesto. Aún en poblados de la costa que tenían mayor acceso a las ciudades, casos más benignos de malaria y otras enfermedades infecciosas, que impedian la intensificación del ritmo de trabajo industrial, eran apenas paliados por las autoridades locales. Solamente 
para algunas regiones del país durante la década de los cuarenta surgió una política gubernamental de erradicación $y$ de control de estas enfermedades.

Uno de los principales obstáculos para combatir la epidemia de La Convención era la ausencia de recursos económicos. Recién en agosto de 1933 el Ministerio de Fomento envió los primeros fondos para combatir la malaria, los cuales durante toda la campaña llegaron a sumar 130,000 soles $^{47}$. Estos recursos provinieron mayoritariamente de los impuestos que gravaban los productos de los valles de $\mathrm{La}$ Convención y Lares que fueron destinados integramente a la campaña sanitaria ${ }^{48}$. Con el objeto de exigir la colaboración económica de los hacendados, el gobierno envió a la región a Eleodoro Camacho, el subdirector de Salubridad.

En el local de la Prefectura del Cusco Camacho se reunió con las autoridades del departamento y los hacendados de La Convención y Lares. En esta reunión los hacendados se comprometieron a colaborar en la campaña, un compromiso que no se cumplió. Asimismo en esta reunión se creó una Dirección General de la Campaña que fue presidida por el Prefecto del Departamento y que incluyó a las autoridades políticas, municipales y religiosas de la región ${ }^{49}$. Es decir, una campaña antimalárica apoyada desde Lima, sólo empezó en octubre de 1933, más de un año después que la epidemia había comenzado.

La resistencia de los hacendados persitio durante toda la campaña, a pesar que el gobiemo había declarado palúdica la región, una disposición que implicaba la asistencia obligatoria de los campesinos por los dueños de

17 Paz Soldán (1933: 9). "Para combatir el paludismo en el Departamento del Cuzco", ES (C) Agosto 4, 1933, p. 4.

48 Estrella Ruiz (1937: 380, 402).

19 "Paludismo en Cuzco, Comisión Ejecutiva para la campaña respectiva", en: Estrella Ruiz (1937: 406). 
las haciendas ${ }^{50}$. En mayo de 1933, el médico titular de La Convención realizo una visita a las haciendas del distrito de Huayopata y comprobó que a pesar que los enfermos llegaban a trescientos, todas las propiedades tenian terrenos pantanosos, ninguna vivienda contaba con telas metálicas o mosquiteros, $y$ algunas haciendas se encontraban completamente abandonadas ${ }^{51}$. Un testimonio de los conflictos entre hacendados y sanitarios fue la crítica que los primeros hicieron a la "superficial" visita de Camacho:

E] Dr. Camacho [...] se dirigió en auto cerrado a Quillabamba donde pudo observar los estragos del mal desde el interior de las vidrieras, mientras se le agasajaba con una champanada morian nueve enfermos sin atención ninguna a pocos pasos. Al día siguiente regresaba al Cusco y hacía creer [...] que el mal estaba conjurado ${ }^{52}$.

Los hacendados se defendieron de las acusaciones de no haber participado activamente en la campaña contra la epidemia arguyendo la ausencia de trabajadores en el valle y culpando a los sanitarios. Según ellos el doctor Geller retenía la quinina enviada desde Lima, vendiéndola a sobreprecio en su botica particular en Quillabamba. Es probable que ello ocurriese, si se toma en cuenta que generalmente las autoridades de Lima toleraban que los médicos complementasen sus ingresos instalando farmacias. Asimismo en un momento en que los

50 "Zonas palúdicas; se declara como tales la provincia de La Convención y el valle de Lares de la provincia de Calca", en: Estrella Ruiz (1937: 339).

51 Médico Sanitario Departamental a Prefecto del Cuzco, Mayo 17, 1933. Prefectura, Comunicaciones Recibidas, Legajo 8, Año 1933, ADC. 
envios de quinina desde Lima tardaban en llegar hasta 20 días, este producto aumentó notoriamente su valor. La quinina fue vendida a precios altos no sólo por los boticarios si no por algunos enfermos que fingían tomarla para más tarde venderla. Más grave aún fue la denuncia de casos en los que la quinina era suplantada por bicarbonato, almidón y otras sustancias tóxicas ${ }^{53}$.

Alentada por los hacendados se presentó una denuncia contra Geller por delitos de concusión y peculado de la quinina. La discusión atravesó la ciudad del Cusco $e$ instituciones populares como la Sociedad de Artesanos de esta ciudad se sumaron a la denuncia ${ }^{54}$. Sin embargo, el Concejo Provincial de Quillabamba desestimó las acusaciones y otorgó a Geller un voto de confianza. El mismo diario El Sol del Cusco intervino en la polémica para defender al médico, señalando que "otros" eran los sanitarios inescrupulosos ${ }^{55}$. Quizás las relaciones cultivadas durante años por el médico provincial, le permitieron salir bien parado de la acusación. Aunque no todos los médicos provinciales se ajustaron a ese rol, la función tradicional de los médicos titulares era la de ofrecer un servicio básicamente urbano, aún en provincias predominantemente rurales, y congraciarsre con las autoridades políticas de la región.

La tensa relación entre sanitarios y hacendados se incrementó por la existencia de diferentes concepciones sobre la curación de la malaria. Reflejando viejas ideas terapéuticas superadas por los conocimientos entonces existentes, los hacendados exigían que se administre a los pacientes un "buen purgante" que les limpie el estóma-

53 Córtez (1938: 50).

54 Ardiles Villegas (1983: 90).

55 "El asunto de la venta de quinina en La Convención", ES (C) Enero 4,1934, p. 2. Camacho también salió en defensa del médico de Quillabamba, "Sobre la supuesta venta de quinina en La Convención", Es (C) Enero 24, 1934, p. 4. 


\section{EPidemia y SOCIEDAd en el Valle de La CONVENCión}

$\mathrm{go}^{56}$. Estas ideas se remontaba al siglo diecinueve cuando se pensaba que la malaria, como muchas otras enfermedades infecciosas y febriles, requerian de un tratamiento antiflogístico consistente en sangría y purgantes. Sin embargo, desde comienzos del siglo XX, había sido demostrado por la ciencia médica que este tipo de tratamiento era totalmente irrelevante, y eventualmente peligroso, para la curación de estas enfermedades.

La campaña también reveló la debilidad de los organismos municipales en una zona dominada por los hacendados. Por ejemplo, el Alcalde de Lares resolvió realizar el drenaje por medio de faenas públicas. Como era dificil convencer a los hacendados para que facilitaran peones para este trabajo, se acordó imponer una fuerte multa a los que incumliesen la ordenanza municipal. No pudiendo cobrar localmente las multas, el Concejo Provincial resolvió que estas fuesen exigidas por la Subprefectura de la provincia y la Prefectura del departamento ${ }^{57}$. Reveladora de esta debilidad fue también la situación del Concejo Provincial de Quillabamba que, en noviembre de 1933, se encontraba en estado acéfalo por la renuncia de sus regidores ${ }^{58}$. De una manera parecida, entre julio $y$ diciembre de 1933, el Concejo de Lares no tenía ningún concejal, quedando sólo el Alcalde a cargo de la municipalidad 59 .

56 "La verdad", p. 2.

57 Finalmente la Prefectura resolvió no aceptar ni cobrar las multas. H. Miranda a Prefecto del Cuzco, Diciembre 7, 1933. Prefectura, Comunicaciones Recibidas, Legajo 8, Año 1933, ADC.

58 Alcalde de La Convención al Prefecto del Cuzco, Noviembre 24, 1933. Prefectura, Comunicaciones Recibidas, Legajo 8, Año 1933, $\mathrm{ADC}$; y H. Valdéz a señor Director de Gobiemo, Diciembre 13, 1933. Ministerio del Interior 331, Cuzco, Prefectura, Año 1933, Archivo General de la Nación.

59 Arístides Muñoz a Prefecto del Cuzco, Febrero, 11, 1933. Prefectura, Comunicaciones Recibidas, Legajo 8, Año 1933, ADC. 


\section{Marcos Cueto}

Además de estos problemas la campaña antimalárica tuvo poca efectividad debido a que las autoridades sanitarias nunca pudieron generalizar el uso de la quinina. En un medio caracterizado por la dispersión de la población, dificilmente la quinina pudo llegar a todas las viviendas. En realidad nunca hubo una verdadera campaña sanitaria en el sentido estricto de la palabra. Lo que ocurrió fue que un médico, 0 un grupo de sanitarios, recorrían el valle de un extremo al otro, atendiendo por pocos dias a quienes se encontraban enfermos sin capacidad de poder vigilar su recuperación. Por ello, los sanitarios fueron denominados popularmente como "repartidores de quinina", ya que muchas veces no hacian nada más que eso ${ }^{60}$. Durante su estadía en el Cusco, Camacho dispuso el control de la distribución de la quinina por medio de carnets que consignaban las cantidades recibidas. Como es de suponer estos carnets no pudieron ser controlados. La falta de vigilancia de este precario sistema se reflejó cuando un médico regresó a una comunidad y encontró que los enfermos no habían tomado las dosis diarias de quinina ${ }^{61}$.

La quinina era rechazada por la población porque se consideraba que una bebida de sabor amargo y cálido no podía servir para combatir una enfermedad cuya manifestación más característica era la fiebre $e^{62}$. Esta idea reflejaba antiguas ideas hipocráticas de origen colonial, que se habían incorporado a la concepción popular de la enfermedad, según la cual una curación debía aplicar lo contrario a lo que se suponía que era la dolencia, es decir, lo frío por lo caliente y viceversa ${ }^{63}$. La quinina era

60 Córtez (1938: 36).

61 Ugarte, "De Sanidad", p. 2.

62 Según el relato de un médico fue necesario obligar a la gente a ingerir por la fuerza la quinina. Luglio Gil (1950: 5).

63 Para una discusión de la antiguiedad y la influencia de la medicina hipocrática en Latinoamérica, véase Foster (1987: 355-393). 


\section{Epidemia y Sociedad en el Valle de La Convención}

rechazada también porque muchos sanitarios administraban dosis normales sin percatarse de los efectos colaterales perniciosos que esta droga podia tener en personas desnutridas ${ }^{64}$.

El uso de mosquiteros y de telas metálicas en la zona fue también de una dudosa efectividad. La mayoría de casas eran de caña y madera, lugares donde los insectos podian entrar y salir fácilmente, independientemente de si las ventanas estuviesen protegidas por telas metálicas o no. El único tipo de construcciones donde la tela metálica podía ser útil era en las de adobe 0 piedra ${ }^{65}$. La miseria en que vivian los propios habitantes impidió que los mosquiteros repartidos se usasen correctamente, puesto que muchas veces estos fueron utilizados como vestido o como abrigo para dormir 66 .

Otro factor que obstaculizó la campaña fue el hecho que la mayoría de los sanitarios no hablaban el quechua o alguna de las otras lenguas nativas de la región ${ }^{67}$. Mayor ignorancia 0 ignorancia 0 indiferencia hubo con respecto a la percepción del campesinado sobre el proceso salud-enfermedad. Para la mayoría de los sanitarios, el principal problema de la campaña no fue la resistencia de los hacendados ni los pocos recursos asignados por el Estado, sino las concepciones populares indigenas. Una queja de los médicos provincial y departamental refleja los prejuicios contenidos en una práctica sanitaria muchas veces enfrentada a la población:

El factor humano es el más grande obstáculo para la divulgación de las nociones elementales de higiene. La generalidad no tiene en absoluto fe en

64 Córtez (1938: 36).

65 Willie (1934: 647).

66 Córtez (1938: 64).

67 lbid., p. 10. Además del quechua y el español las lenguas más importantes de la región eran el machiguenga, el huarayo, el arasairi y el mashco, Menendez Rua (1948: 137). 


\section{Marcos Cueto}

los progresos de la medicina prefiriendo la intervención de los curanderos empíricos y brujos, cuyas prácticas se hallan llevadas al absurdo ${ }^{68}$.

No hubo un esfuerzo por entender que los campesinos percibían la salud y la enfermedad dentro de una visión global de la naturaleza, a la que consideraban profundamente espiritualizada ${ }^{69}$. Para el campesino, la medicina popular indígena era un sistema de creencias con una ventaja sobre la medicina occidental: suponía una visión moral del mundo ${ }^{70}$. Ciertas ceremonias y costumbres mantenían la salud, fuerzas sobrenaturales castigaban con la enfermedad a los que no las seguian, y los hechizos podian manipular estas fuerzas. Reconciliarse con el ritual incumplido o evadir el hechizo de un enemigo parecían terapias que iban al fondo del problema, a diferencia de la medicina occidental que se concentraba en los sintomas.

Durante la epidemia en La Convención no se intentó una articulación entre los recursos sanitarios indigenas y los métodos de la medicina occidental $y$, por el contrario, se acentuó la tensión entre las concepciones mágicas y científicas de la enfermedad. Algunos ejemplos ocurridos en Puno a mediados de los años treinta demuestran que era posible rodear el conflicto, buscando una asimilación exitosa de la terapia occidental en las concepciones folklóricas ${ }^{71}$. Esta última por tener cierta base empírica podría haber asumido estos elementos sin poner en peligro sus concepciones globales.

Tampoco se pensó que después de años de abandono sanitario era imposible desplazar abruptamente las concepciones populares y a los practicantes informales

68 Ugarte y Geller (1933: 529).

69 Al respecto véase Zavaleta Arteaga (1946).

70 Aguiló (1985).

71 El autor está haciendo un estudio sobre la labor realizada por el médico "indigenista" Manuel Nuñez Butrón. 
de la medicina que tradicionalmente habían lidiado con la enfermedad entre la población indígena. La intolerancia ante las prácticas altemativas de la medicina se explica porque en ese momento la medicina oficial peruana estaba empeñada en monopolizar el cuidado profesional de la salud, eliminando a curanderos, herbolarios y charlatanes. Este objetivo sólo se consiguió muy parcialmente algunos años después en algunas ciudades ${ }^{72}$.

La incapacidad para revalorar la medicina popular indígena era congruente con la marginación cultural y la sobreexplotación a que estaban sometidos los campesinos. Cualquier otra actitud de los sanitarios hubiese significado cuestionar la "superioridad" de la cultura de los propietarios. Por ello, en última instancia, los hacendados y las autoridades sanitarias de Lima concordaban en que las medidas debían ser impuestas autoritariamente, basándose principalmente en los recursos tecnológicos, independientemente de su adaptabilidad al medio. Las discusiones entre hacendados y sanitarios puede entenderse no sólo como una evasión de responsabilidades, sino como disputa sobre distintos métodos que finalmente estaban dirigidos a un fin común: mantener el control de la población durante la crisis social provocada por la epidemia.

En medio de la resistencia de los hacendados, los cuestionamientos a los médicos y la incredulidad de los indígenas, la malaria siguió avanzando hasta abril de 1934, cuando concluyó no como resultado de alguna acción humana sino más bien después de eliminar $e$ infectar a toda la población susceptible al mal. Para entonces la campaña antimalárica en los valles se había reducido a una labor lenitiva, es decir, ni siquiera curativa o asistencial. Según los padres dominicos sólo una quinta parte de la población fue atendida durante la epidemia ${ }^{73}$.

72 Al respecto véase, Cueto (1989: 84).

73 Anónimo (1934: 27). 
Las autoridades sanitarias calcularon en 6,000 las personas que murieron $y$ en 15,000 las que enfermaron de malaria duante la epidemia ${ }^{74}$. Para una población de 25,000 habitantes, éste era un número altísimo ${ }^{75}$. Según otro cálculo murieron muchos más, entre 8,000 y 10,000 personas ${ }^{76}$. Es difícil establecer una cifra exacta, en un lugar donde cualquier lugar era apropiado para enterrar a los muertos y no existian registros públicos adecuados. Sin embargo, según varios estudios históricos, esta constituyó una verdadera catástrofe demográfica de la que la región tardó en recuperarse ${ }^{77}$.

Después de esta epidemia, la malaria se volvió endémica en la provincia. La carencia de recursos médicos empezó a ser resuelta parcialmente en 1938 cuando se acabó de construir el Hospital en Quillabamba. Sin embargo, sólo en la década del cuarenta a través de campañas masivas de erradicación de mosquitos con insecticidas de acción residual como el DDT, organizadas por las Naciones Unidas y el Ministerio de Salud del Perú, se redujo considerablemente la prevalencia de malaria en la zona.

La epidemia que atacó a fines de 1932 el valle de La Convención reveló las carencias de una colonización con métodos primitivos, donde todo el poder radicaba en hacendados que no estaban dispuestos a pagar el costo de una verdadera modernización. Fue asimismo un episodio que produjo abundantes testimonios sobre las miserables condiciones de vida de los campesinos, la precariedad de las instituciones políticas y las insuficiencias de los sistemas oficiales de salud pública en los Andes. Más

1933, p. 2.

74 "Oficio del Subdirector de Salubridad", ES (C) Setiembre 5,

75 Donayre Rojas (1947: 7).

76 Luglio Gil (1950: 3).

77 Véase por ejemplo, Hobsbawm (1969: 33-34); y Fioravanti (1976: 57-58). 


\section{Epidemia y SOCIedad en el Valle de La Convención}

reveladora aún fue la evidencia de las profundas diferencias culturales que separaban a los pobladores sobre hechos tan cotidianos como la salud y la enfermedad. La epidemia no sólo mostró estas diferencias si no que las enfrentó de una manera en que la población indígena ganó muy poco. 


\section{Bibliografía}

ACKERKNECHT, E.

1965 History and geography of the most important diseases, Hafner Publishing Company, Nueva York.

AGUILO, $F$.

1985 Enfermedad y Salud, según la concepción Aymaro-Quechua, Talleres Gráficos Qori Llama. Sucre.

ARDILES VILLEGAS, T.

1983 "Apuntes autobiográficos de un colonizador", en: Marco Aurelio Ugarte Ochoa, La Convención 81-88.

\section{ANÓNIMO}

1933 "Editorial", Revista Médica, 172:172.

ANÓNIMO

1934

"De la Convención", Misiones Dominicanas del Perú, 16:80-81.

ARAGÓN SARAVIA, C.

1941 "La Malaria en los valles de La Convención y Lares, sector Echerate, 1936-1939", 


\section{Biblografla}

Tesis de Bachiller, Universidad de San Marcos, Lima.

BRISSEAU, J.

1983

"El papel del Cusco en la colonización de la ceja de montaña y de la montaña", en: Ugarte Ochoa, La Convención, 15-34.

BRUCE-CHWARR, L.J.

1985 Essential malariology, Division John Willey \& Sons, Nueva York.

COOK, N.D.

1981 Demographic Collapse, Indian Peru 15201520, Cambridge University Press, Cambridge.

CORTEZ, M.

1938 "Epidemia palúdica de los valles de La Convención y Lares", Tesis de Bachiller, Universidad de San Marcos, Lima.

CUADROS, F.

1949

El arriendo y la reforma agraria en la provincia de La Convención, H.G. Rosas, Cusco.

CUETO, $M$.

1989 Excelencia científica en la periferia: Actividades científicas e investigación biomédica en el Perú 1890-1950, Tarea, Lima.

DOBYNS, H.F.

1963 "An outline of Andean Epidemic History to 1720", Bulletin of the History of Medicine, 37, 6: 493-515. 


\section{Marcos Cueto}

DONAYRE ROJAS, R.

1947 "Contribución al estudio entomológico de la malaria en el Perú, Departamento del Cusco, Valle de La Convención", Tesis de Bachiller, Universidad de San Marcos, $\mathrm{Li}$ ma.

ESTRELLA RUIZ, J.A.

1937 Prontuario de la Legislación Sanitaria del Perú, Tomo III, Imprenta Americana, Lima.

FIORAVANTI, E.

1976 Latifundio y Sindicalismo Agrario en el Perú, Instituto de Estudios Peruanos, Lima.

FLOREZ MUÑIZ, G.

1946 "Organización social, religiosa y económica en una hacienda del valle de Lares", Monografías, Universidad San Antonio Abad, Vol. 2, Archivo Departamental del Cusco.

FOSTER, G.

1987 "On the origin of Humoral Medicine in Latin America", Medical Anthropology Quarterly, $1,4: 355-393$.

HARRISON, G.

1978 Mosquitoes, malaria and man: a history of the hostilities since 1880, E.P. Dalton, New York.

HERMOZA, E.

1944 "El paludismo y sus consecuencias en el valle de Lares", Monografías, Universidad San Antonio Abad, vol. 3, Archivo Departamental del Cusco. 


\section{BiBLIOGRAFIA}

HERZLCH, C. y PIERRET, J.

1987 Ilness and self in society, The Johns Hopkins University Press, Baltimore.

HOBSBAWM, E.

1969

"A Case of Neo-Feudalism: La Convencion Peru", Journal of Latin American Studies, 1,1: 31-50.

JACOBSEN, $\mathrm{N}$.

1983

"El caso de la economía ganadera en el sur peruano, 1855-1920", Allpanchis, 18, 21 : 89-148.

KUCZYNSKI GODARD, M.

1945 Estudios médicos de la región sur del Perú. Ministerio de Salud Público y Asistencia Social, Lima.

LUGLIO GL, $M$.

1950 "Campaña antimalárica en La Convención y Yanatile", Tesis de Bachiller, Universidad de San Marcos, Lima.

MCKEOWN, T.

1976 El papel de la medicina, ¿Sueño, espejismo o nimesis? Siglo XXI, México.

MENENDEZ RUA, A.

1948 Paso a la Civilización, obra histórica en la que se estudia el aspecto religioso y su desenuolvimiento en la provincia de La Convención, Sanmarti, Lima.

MOSCOSO, A.

1944 "Arriendos del valle de La Convención", Monografias, Universidad San Antonio Abad, Vol. 3, Archivo Departamental del Cusco. 
Marcos Cueto

PAZ SOLDÁN, C.E.

1933 La pandemia malárica en los valles cálidos del Cusco, Instituto de Medicina Social, Lima.

PAZ SOLDÁN, M.F.

1877 Diccionario geografico, estadístico del Perú, Imprenta del Estado, Lima.

PÉREZ, R.

1938 "Estudio social, científico e historia de la campaña antipalúdica en los valles de Lares y La Convención", Tesis de Bachiller, Universidad de San Marcos, Lima.

SLACK, P.

1985

The impact of plague in Tudor and Stuart England, Routledge and Kegan Paul, Boston.

TAMAYO HERRERA, J.

1980 Historia Social del Cusco Republicano, Editorial Universo, Lima.

UGARTE, L. Y GELLER, S.

1933 "La opinión de los médicos sanitario departamental y titular de La Convención sobre la pandemia malárica de las tierras cálidas del Cusco", Reforma Médica, 177: 508530 .

UGARTE Ochoa, M.A.

1983 La Convención, el trabajo y sus luchas sociales, Imprenta Amauta, Cusco.

VALENCIA VARGAS, $F$.

1961 Algunos aspectos del folklore de La Convención, Editorial H.G. Rozas, Cusco. 
Epidemia y Sociedad en el Valle de la Convención

VERGARA, E.

1947 "La trayectoria de la educación en la Provincia de La Convención", Monografías, Universidad San Antonio Abad, Vol. 7, Archivo Departamento del Cusco.

WILLE, J.

1934

"La fauna anofelina malarigena de las zonas cálidas del Cusco", Revista Médica, 196: 646-652, Lima.

YOUNG, M.D.

1976 "Malaria", en: George Hunter, J. Clyde Swartzwelder y David Clyde, Tropical Medicine W.B. Saunders Company, Philadelphia: 353-396.

ZAVALETA ARTEAGA, T.

1946 "La medicina de los antiguos habitantes del Perú", Monografía, Universidad San Antonio de Abad, Vol. 6, Archivo Departamental del Cusco. 